\title{
V.P. Goryachkin's rational equation in differential form
}

\author{
Eduard Zhalnin ${ }^{1, *}$ \\ ${ }^{1}$ Federal Research Agro-Engineering Center VIM, 1st Institutskiy proezd, 5, Moscow, 109428, Russia
}

\begin{abstract}
The so-called V.P. Goryachkin's rational equation is commonly used to calculate plow drag value and estimate the pulling force required during tilling in the field. Its first component represents drag value of the plow mass multiplied by the friction ratio (rolling friction, sliding friction) of the plow elements against the furrow bottom and walls. The second component describes the parameters of soil layer tilled and the ratios describing the type and condition of the soil. The third element describes the soil layer parameters, plow speed squared and energy ratio, which reflects the soil characteristics, type and condition of the plow surfaces rubbing against the soil and the soil lifting force. The rational equation has been tested in numerous experiments and proved satisfactory in most cases, with different plows and different soil types; the margin of error varies, however. Moreover, the formula proved to be universal and found use in other cases to calculate the drag of a certain object in liquid or gaseous environments. In this work the authors derive a general system of differential equations which can be solved to derive V.P. Goryachkin's rational equation and its numerous variants and specific applications.
\end{abstract}

\section{Introduction}

V.P. Goryachkin's equation is based not on strict mathematical calculations but on the general principles of rationality and practicability, as any complex process can always be represented as a sum total of its simple elements.

The classical equation to calculate plow pulling force, or the so-called V.P. Goryachkin's rational equation, looks as follows [1]:

$$
P=f G+К а в+\varepsilon a \text { в } V
$$

where $f$ is the friction ratio between outer surfaces of the plow (plow leg, plow share, plow breast, etc.) and the furrow walls and bottom;

$\mathrm{G}$ - is the plow mass;

$\mathrm{K}$ - is the soil resistance to deformation ratio;

$a$ - is the tilling depth (height of the soil layer tilled);

8 - is the width of the soil layer;

$\varepsilon$ - is the energy change ratio of soil particles when thrown aside by the plow breast;

$\mathrm{V}$ - is the speed of the plow in the direction of travel.

*Corresponding author: zhalnin@yandex.ru 
V.P. Goryachkin himself called this equation rational (or reasonable, advisable). The equation takes the form of a trinomial, which logically combines three factors: the working body parameters, the environment (soil) parameters and the dynamics of their interaction process. V.P. Goryachkin represented the complex process of plow drag during tilling as a sum total of three components, which fully correspond to the physical process of plow operation. The first component is the plow mass and friction ratio of its primary services interacting with the soil layer being tilled. There is nothing theoretical about this component, as it represents the ordinary and well known physical law of pressure drag exhibited by any object that has to be moved from an idle position: the force resulting from the mass of the body has to be multiplied by the friction ratio against the bearing surface.

The second component in the equation is the contact surface of the working body with the environment, i.e. the plow elements contacting the soil layer being tilled. The author adds soil resistance to deformation ratio, or $\mathrm{K}$. This is also quite a natural component, its nature and unit of measurement (e.g., $\mathrm{N} / \mathrm{cm}^{2}$ ) being understood from its physical concept.

The third component is also a natural one, as it is well known that the drag of any moving object is dependent on its speed. At lower speeds, less than 1-1.2 m/s, the drag ratio is negligible, which is what V.P. Goryachkin does when measuring the drag of a horsedrawn plow. At medium speeds, approximately $2-8 \mathrm{~m} / \mathrm{s}$, the drag force is the function of speed squared, and at speeds of more than $8 \mathrm{~m} / \mathrm{s}$ - of speed raised to the third power. Since tractor-drawn agricultural machinery operates in the second speed range, the rational formula (1) uses plow speed squared.

Thus, without going into complex mathematical calculations, based solely on wellknown physical laws, V.P. Goryachkin successfully used logic to derive the most reasonable, universal and rational equation to calculate drag force for plow and similar technical equipment. But that is what the talent of a great scientist is about - being able to use simple logical reasoning to arrive to a very important general physical law. V.P. Goryachkin's rational equation proved the most adequate way to approximate the experimental data on plow pulling force.

Moreover, it often revealed errors in experiments, and deviations from the experimental data were in most cases found to be caused by inaccuracies in measuring the $\kappa$, a, в, $\varepsilon$ parameters, rather than the defects in the formula itself. V.P. Goryachkin did indeed arrive to his classical equation in a logical way.

In this regard, we became interested if it is possible to derive the same classical equation by using purely mathematical methods, e.g., differential equations.

Purpose of the study. Produce V.P. Goryachkin's rational equation by using differential equations.

\section{Materials and methods}

The analysis is based on logical and conceptual notions used by V.P. Goryachkin to develop his rational equation to calculate drag. The methodological basis for the study is represented by the basic notions of differential equations and axiomatic function tables [2].

\section{Results and discussion}

Three parameters are taken as source data: $\mathrm{G}-$ the plow mass, $\mathrm{F}=\mathrm{a} \cdot \mathrm{B}-$ cross-section area of the soil layer tilled and $\mathrm{V}$ - the plow speed.

Based on a priori axiomatic reasoning, let's accept that increase in plow drag $\Delta \mathrm{P}_{1}$ to increase in soil layer area is a constant value, i.e. the drag increases with the increase in the area of soil layer tilled, which is reasonable for plow parameters used in practice. 
Then the first differential equation looks as follows:

$$
\frac{d P_{1}}{d F}=K
$$

Integral solution to equation (2):

$$
P 1=K F+C_{1}
$$

The constant of integration $-\mathrm{C}_{1}-$ is determined by the classical method, i.e. at $\mathrm{F}=0$. Then $\mathrm{C}_{1}=\mathrm{P}_{1}$. Based on the physical essence of the tilling process and equation (3), $\mathrm{C}_{1}$ can be represented in absence of soil layer $(\mathrm{F}=0)$ as the product of plow mass and the friction ratio of plow parts in contact with the soil.

$$
C_{1}=P_{1}=f G
$$

After which equation (3) can be recorded as follows:

$$
\mathrm{P} 1=\mathrm{fG}+\mathrm{KF}
$$

The component of plow drag depending on the speed per area unit of soil layer tilled in differential form can be determined from the following differential equation:

$$
\frac{d P_{2}}{F d V}=\varepsilon
$$

The integrated solution to equation (6) looks as follows:

$$
P_{2}=\varepsilon F V^{2}+C_{2}
$$

The constant of integration will be determined at $\mathrm{V}_{\mathrm{o}}=0$ and $\mathrm{F}=0$

$$
C_{2}=P_{2}=C_{1}=P_{1}
$$

By substituting the value of $\mathrm{C}_{2}=\mathrm{P}_{1}$ in equation (7), we get the total plow drag as a function of $\varepsilon, \mathrm{F}$ and $\mathrm{V}$ :

$$
P_{o}=f G+K F+\varepsilon F V^{2}
$$

Or, in the more traditional format:

$$
P_{o}=f G+k \cdot a \cdot b+\varepsilon a \cdot \theta V^{2}
$$

Thus, the V.P. Goryachkin Rational Equation can be derived by solving a system of two differential equations and calculating one integrated value:

$$
\left\{\begin{array}{c}
\frac{d \mathrm{P}_{1}}{\alpha F}=K \\
\frac{d \mathrm{P}_{2}}{F \cdot \alpha V}=\varepsilon \\
P_{1}=P_{1}+P_{2}
\end{array}\right.
$$


The first equation determines the pulling force as a function to the area of soil layer tilled, the second one - as a function to plow speed, and the third one represents the sum total of integral solutions of the first two equations.

\section{References}

1. V.P. Goryachkin A rational formula for the thrust force of plows (Moscow: Ed. in the Central press Office of the USSR Supreme Soviet, 1925)

2. E.V. Zhalnin Axiomatization of agricultural mechanics. (Moscow: VIM, 2001) 